\title{
Novel local learning rule for neural adaptation fits Hopfield memory networks efficiently and optimally
}

\author{
Chris Hillar*, Jascha Sohl-Dickstein, Kilian Koepsell \\ From Twenty Second Annual Computational Neuroscience Meeting: CNS*2013 \\ Paris, France. 13-18 July 2013
}

We present an algorithm to store binary memories in a Little-Hopfield neural network using minimum probability flow, a recent technique to fit parameters in energy-based probabilistic models. For memories without noise, our algorithm provably achieves optimal pattern storage and outperforms classical methods both in speed and memory recovery. Moreover, when trained on noisy or corrupted versions of a fixed set of binary patterns, our algorithm finds networks which correctly store the originals. We also demonstrate this finding visually with the unsupervised storage and clean-up of large binary fingerprint images from significantly corrupted samples.

\section{Background}

In 1982, motivated by neural modeling work of [3] and the Ising spin glass model from statistical physics [2], Hopfield introduced a method for the storage and retrieval of binary patterns in an auto-associative neural-network [1]. However, existing techniques for training Little-Hopfield networks suffer either from limited pattern capacity or

\begin{tabular}{|c|c|c|c|}
\hline & $\begin{array}{l}\text { Outer Product } \\
\text { Rule (OPR) }\end{array}$ & $\begin{array}{l}\text { Perceptron } \\
\text { (PER) }\end{array}$ & $\begin{array}{c}\text { Minimum } \\
\text { Probability Flow } \\
\text { (MPF) }\end{array}$ \\
\hline Patterns Storable & $N /[4 \log (N)]$ & $1.6 \mathrm{~N}$ & $1.6 \mathrm{~N}$ \\
\hline Optimal & NO & YES & YES \\
\hline Tractable & YES & NO & YES \\
\hline Local Learning Rule & YES & YES & YES \\
\hline Learns from Noisy Patterns & NO & NO & YES \\
\hline Cleanup / Recovery & Poor & Better & Best \\
\hline
\end{tabular}

Figure 1 Performance. Comparison of our training algorithm (MPF) to classical methods Outer Product Rule (OPR) and Perceptron (PER).

* Correspondence: chillar@msri.org

Redwood Center for Theoretical Neuroscience, Berkeley, CA 94720, USA B-1 Ced Central Attribution License (http://creativecommons.org/licenses/by/2.0), which permits unrestricted use, distribution, and reproduction in any medium, provided the original work is properly cited. 


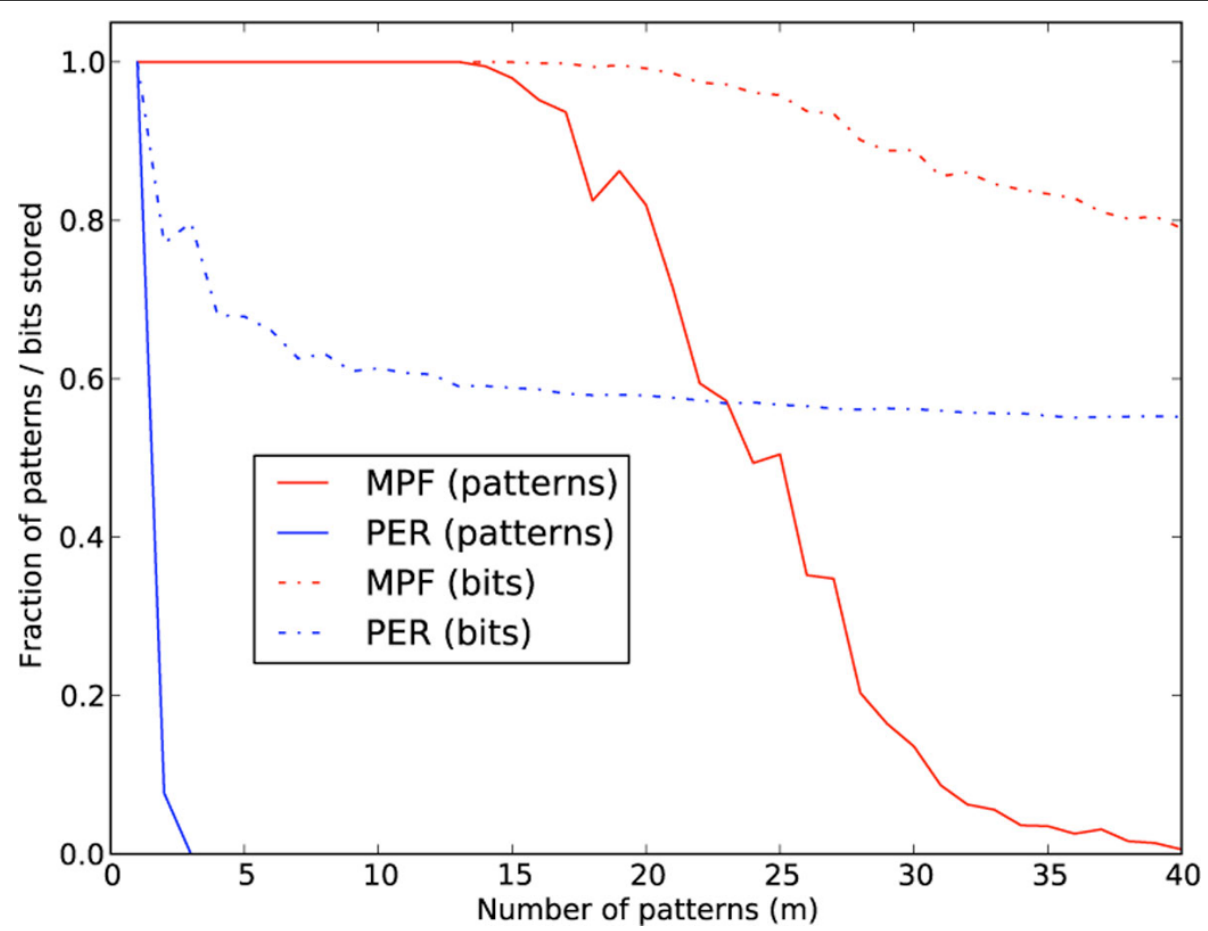

Figure 2 Noisy data storage. Shows fraction of patterns (red for MPF, blue for PER) and fraction of bits (dotted red for MPF, dotted blue for PER) recalled of trained networks ( $n=64$ nodes each) as a function of the number of patterns $m$ to be stored. Training patterns were presented repeatedly with 20 bit corruption (i.e., $31 \%$ of the bits flipped).

excessive training time, and they exhibit poor performance when trained on unlabeled, corrupted memories.

\section{Results}

Our main theoretical contributions here are the introduction of a tractable and neurally-plausible algorithm MPF for the optimal storage of patterns in a Little-Hopfield network, a proof that the capacity of such a network is at least one pattern per neuron, and a novel local learning rule for training neural networks. Our approach is inspired by minimum probability flow [4], which fits probabilistic models without computations with a partition function.

We also present several experimental results. Compared with standard techniques for Little-Hopfield pattern storage, our method is shown to be superior in efficiency and generalization (Figure 1). Another finding is that our algorithm can store many patterns in a LittleHopfield network from highly corrupted (unlabeled) samples of them (Figure 2). We also store $64 \times 64$ binary images of human fingerprints from highly corrupted versions (Figure not shown).

Published: 8 July 2013

\section{References}

1. Hopfield JJ: Neural networks and physical systems with emergent collective computational abilities. Proc Natl Acad Sci U S A 1982, 79:2554-2558.
2. Ising E: Beitrag zur Theorie des Ferromagnetismus. Zeitschrift fur Physik $1925,31$.

3. Little WA: The existence of persistent states in the brain. Math Biosciences 1974, 19.

4. Sohl-Dickstein J, Battaglino P, DeWeese MR: Minimum probability flow learning. PRL 2011.

doi:10.1186/1471-2202-14-S1-P215

Cite this article as: Hillar et al:: Novel local learning rule for neural adaptation fits Hopfield memory networks efficiently and optimally. BMC Neuroscience 2013 14(Suppl 1):P215.
Submit your next manuscript to BioMed Central and take full advantage of:

- Convenient online submission

- Thorough peer review

- No space constraints or color figure charges

- Immediate publication on acceptance

- Inclusion in PubMed, CAS, Scopus and Google Scholar

- Research which is freely available for redistribution

Submit your manuscript at www.biomedcentral.com/submit
C Biomed Central 